Provided for non-commercial research and education use. Not for reproduction, distribution or commercial use.



This article appeared in a journal published by Elsevier. The attached copy is furnished to the author for internal non-commercial research and education use, including for instruction at the authors institution and sharing with colleagues.

Other uses, including reproduction and distribution, or selling or licensing copies, or posting to personal, institutional or third party websites are prohibited.

In most cases authors are permitted to post their version of the article (e.g. in Word or Tex form) to their personal website or institutional repository. Authors requiring further information regarding Elsevier's archiving and manuscript policies are encouraged to visit:

http://www.elsevier.com/copyright 


\title{
VUV spectroscopy of wide bandgap materials
}

\author{
Andrzej J. Wojtowicz \\ Instytut Fizyki, Uniwersytet M. Kopernika, ul. Grudziądzka 5, 87-100 Torun, Poland
}

\section{A R T I C L E I N F O}

\section{Article history:}

Received 8 September 2008

Accepted 10 December 2008

Available online 5 May 2009

\section{PACS:}

29.40.Mc

71.20.Ps

71.35. $-\mathrm{y}$

78.55. Hx

78.60.-b

Keywords:

$\mathrm{BaF}_{2}: \mathrm{Ce}$

$\mathrm{BaF}_{2}: \mathrm{Er}$

(Ba,La)F $\mathrm{F}_{2}$ :Er

UV and VUV spectroscopy

$\mathrm{Er}^{3+}, \mathrm{Ce}^{3+}$ ions

\begin{abstract}
A B S T R A C T
In this paper we will present VUV spectroscopy experiments performed at the Superlumi station of Hasylab, DESY, Hamburg, on samples of $\mathrm{BaF}_{2}$ crystals activated with $\mathrm{Ce}$ and $\mathrm{BaF}_{2}$, $(\mathrm{Ba}, \mathrm{La}) \mathrm{F}_{2}$ crystals activated with Er. The results of these experiments include time resolved luminescence and luminescence excitation spectra obtained under wavelength selective VUV and UV excitation by pulsed synchrotron radiation.

We will reveal the information provided by the VUV/UV excitation spectra of the $\mathrm{Ce}^{3+} 5 \mathrm{~d} \rightarrow 4 \mathrm{f}$ as well as $\mathrm{Er}^{3+} 4 \mathrm{f}^{n-1} 5 \mathrm{~d} \rightarrow 4 \mathrm{f}^{n}$ and $4 \mathrm{f}^{\mathrm{n}} \rightarrow 4 \mathrm{f}^{n}$ emissions on energy transfer mechanisms from the fluoride host to the rare earth ion. We will demonstrate that the fast energy transfer channels involve bound excitons while the generation of free electrons and holes leads to slower processes dependant on hole and/or electron trapping.

We will demonstrate that differences between the excitation spectra of the $5 \mathrm{~d} \rightarrow 4 \mathrm{f}$ emission in Ce and $4 \mathrm{f}^{10} 5 \mathrm{~d} \rightarrow 4 \mathrm{f}^{11}$ emission in Er activated $\mathrm{BaF}_{2}$ are generated by the coupling of the $4 \mathrm{f} \rightarrow 5 \mathrm{~d}$ transition to the $4 \mathrm{f}^{10}$ core of the $\mathrm{Er}^{3+}$ ion. We will also identify the additional band, absent for Ce, which is due to the exchange split high spin (HS) state of the $4 \mathrm{f}^{10} 5 \mathrm{~d}$ configuration responsible for the slow decay of the excited $\mathrm{Er}^{3+}$ ions in $\mathrm{BaF}_{2}$ and $(\mathrm{Ba}, \mathrm{La}) \mathrm{F}_{2}$.

Finally we will provide evidence and explain why the dominant VUV $4 \mathrm{f}^{10} 5 \mathrm{~d} \rightarrow 4 \mathrm{f}^{11} \mathrm{Er}^{3+}$ emission in $\mathrm{BaF}_{2}$ is spin-forbidden and slow while in the mixed $(\mathrm{Ba}, \mathrm{La}) \mathrm{F}_{2}$ crystals it is spin-allowed and fast.
\end{abstract}

(C) 2009 Elsevier B.V. All rights reserved.

\section{Introduction}

The surge of interest in VUV spectroscopy of solid state materials is largely due to new and demanding applications such as UV and VUV solid state lasers, fast and efficient scintillators and new "quantum-cutting" phosphors driven by mercury-free discharge radiation. At the forefront of materials considered for these attractive new applications are wide bandgap materials activated with rare earth ions.

An important additional incentive is provided by disparity between easily accessible model programs providing energies for a large number of emission and absorption lines of rare earth ions and scarce experimental results especially in the UV and VUV spectral ranges. We note that the so-called "extended Dieke's" diagram based on model calculations partly verified by experiment and covering energies above $40,000 \mathrm{~cm}^{-1}$ has been established only recently $[1,2]$. Experimental verification for some of these energies is still missing [3].

We also note that the VUV excitation spectra of rare earth emissions reflect various mechanisms of energy transfer from the host to the ion enabling evaluation of e.g. scintillator materials. The

E-mail address: andywojt@fizyka.umk.pl annual Hasylab reports published on-line provide numerous examples, see e.g. ${ }^{1}$.

In this paper we report experimental studies of UV and VUV luminescence from $\mathrm{Ce}$ and $\mathrm{Er}$ activated crystals of $\mathrm{BaF}_{2}$ and Er activated mixed crystals of $(\mathrm{Ba}, \mathrm{La}) \mathrm{F}_{2}$ using wavelength selective, pulsed synchrotron radiation. The results that we have obtained confirm and extend earlier studies of other fluorides [3], as well as provide some new experimental observations, such as fast and efficient VUV emission from $(\mathrm{Ba}, \mathrm{La}) \mathrm{F}_{2}:$ Er.

\section{Crystals and experimental set-ups}

The $\mathrm{Ce}$ and $\mathrm{Er}$ doped samples of $\mathrm{BaF}_{2}$ and $(\mathrm{Ba}, \mathrm{La}) \mathrm{F}_{2}$ were cut from larger boules grown by Optovac Inc. (North Brookfield, MA, USA) using the Bridgman method. The concentration of $\mathrm{Ce}$ in the melt was 0.015 and $0.05 \mathrm{~mol} \%$ for the Ce-doped boules of $\mathrm{BaF}_{2}$. The $0.05 \mathrm{~mol} \%$ boule was co-doped with $\mathrm{Na}(0.2 \mathrm{~mol} \%)$ to minimize interstitial fluorine compensation. The $\mathrm{Er}$ concentrations in $\mathrm{Er}$ doped boules of $\mathrm{BaF}_{2}$ were 0.05 and $0.2 \mathrm{~mol} \%$ in the melt. The $(\mathrm{Ba}, \mathrm{La}) \mathrm{F}_{2}$ boule contained $30 \mathrm{~mol} \%$ of La and $0.2 \mathrm{~mol} \%$ of Er. The samples were not subjected to any chemical reducing procedure. They

\footnotetext{
${ }^{1}$ http://www.fizyka.umk.pl/andywojt/physics.htm.
} 


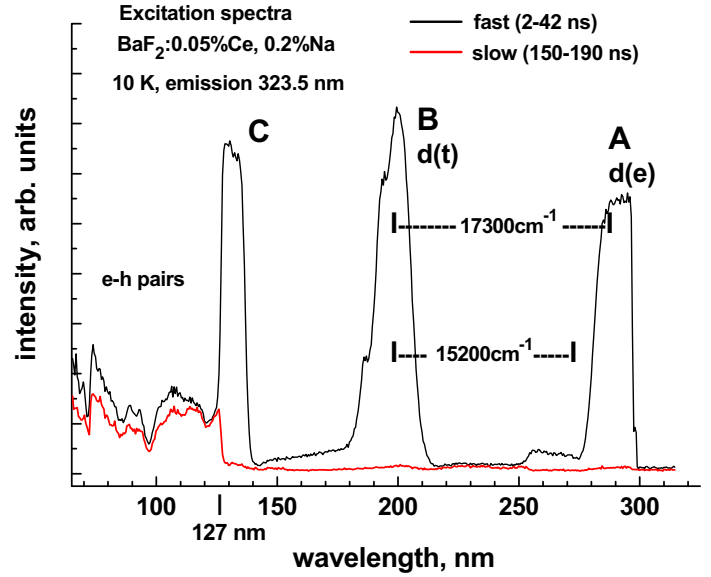

Fig. 1. Time resolved excitation spectra of Ce-emission in $\mathrm{BaF}_{2}: 0.05 \mathrm{~mol} \% \mathrm{Ce}$, $0.2 \mathrm{~mol} \% \mathrm{Na}$ at $10 \mathrm{~K}$. The spectra have been corrected using a salicylate standard. Resolution was $0.32 \mathrm{~nm}$, step $0.5 \mathrm{~nm}$. Black line, signal accumulated in the time window set at 2-42 ns ("fast" spectrum), red line, 150-190 ns ("slow" spectrum). For details see text. (For interpretation of the references to colour in this figure legend, the reader is referred to the web version of this article.)

were of high optical quality, clear, displayed no color, no inclusions and no indication of oxygen contamination.

The VUV/UV experiments (luminescence and excitation spectra) were performed at the SUPERLUMI station of HASYLAB on the Doris III storage ring in DESY, Hamburg, Germany. A detailed description of SUPERLUMI's experimental facilities, available online $^{2}$, was also given by Zimmerer [4].

For excitation we have used a primary $2 \mathrm{~m}$ normal incidence monochromator in $15^{\circ}$ McPherson mounting, equipped with the holographic concave grating (1200 groves $/ \mathrm{mm})$, coated by $\mathrm{Al}+\mathrm{MgF}_{2}(50-330 \mathrm{~nm})$. The resolution was $0.32 \mathrm{~nm}$.

For emission we have used two monochromators: the homemade $0.5 \mathrm{~m}$ Pouey VUV monochromator (f/2.8, fixed resolution of $1.1 \mathrm{~nm}$ ), equipped with the solar blind Hamamatsu R6836 photomultiplier (115-300 nm) for VUV, and the Acton Research $0.3 \mathrm{~m}$ Czerny-Turner monochromator "Spectra Pro 300i" (f/4) equipped with the Hamamatsu R6358P photomultiplier for longer wavelengths spectra (200-800 nm). Resolution of this monochromator depended on the adjustable slit width and the grating inserted. We have used three gratings, one having $1200 \mathrm{~g} / \mathrm{mm}(2.7 \mathrm{~nm} /$ $\mathrm{mm}$ ) and blazed at $300 \mathrm{~nm}$ and two others having $300 \mathrm{~g} / \mathrm{mm}$ $(10.8 \mathrm{~nm} / \mathrm{mm})$ and blazed at 300 and $500 \mathrm{~nm}$, respectively.

\section{Experimental results and discussion}

In Fig. 1 we present time resolved excitation spectra of the Ce emission ( $323.5 \mathrm{~nm}$ ) measured at $10 \mathrm{~K}$ from the sample of $\mathrm{BaF}_{2}$ : activated with $\mathrm{Ce}$ and $\mathrm{Na}(0.05 \mathrm{~mol} \% \mathrm{Ce}, 0.2 \mathrm{~mol} \% \mathrm{Na})$. The spectra have been corrected for spectral sensitivity of the set-up using the salicylate standard. The time window used to integrate emission photon counts was set at 40 ns and delay was set at 2 ns for the "fast" spectrum (black solid line) and 150 ns for the "slow" spectrum (red solid line). The "fast" spectrum shows three main bands, labeled A, B and C. The peak position of the A-band $(5 \mathrm{~d}(\mathrm{e}))$ at $291 \mathrm{~nm}$ as well as peak position and the structure of the B-band $(5 \mathrm{~d}(\mathrm{t}))$ at about $200 \mathrm{~nm}$ are no different that in the corresponding spectrum of the lower Ce concentration (0.015 mol\%Ce) sample (not shown) and numerous spectra measured earlier (compare e.g. [5]). We note that positions of the spectrally resolved subbands of the B-band at about 186.5, 194.0 and

\footnotetext{
${ }^{2}$ http://hasylab.desy.de/facilities/doris_iii/beamlines/i_superlumi/index_eng.html.
}

$199.9 \mathrm{~nm}$, suggest that $10 \mathrm{Dq}$, at about $17,300 \mathrm{~cm}^{-1}$, is much larger than energies associated with the lower symmetry crystal field component.

Somewhat lower value of $10 \mathrm{Dq}$ (at $15,200 \mathrm{~cm}^{-1}$ ) is obtained if one assumes that the weak broad band at about $265 \mathrm{~nm}$ is due to the forbidden transition to the $5 \mathrm{~d}(\mathrm{e})$ sublevel split off by the combined action of spin-orbit interaction and lower symmetry crystal field component [6]. We note in passing that $17,300 \mathrm{~cm}^{-1}$ value is close to the value of $17,400 \mathrm{~cm}^{-1}$ obtained for $\mathrm{Ce}$ in $\mathrm{CaF}_{2}$ by Manthey, who analyzed positions of all d-bands to fully characterize the crystal field [6].

In any case it seems reasonable to assume that in both Na-codoped and Na-free crystals we are dealing with roughly the same dominant "effective" Ce site of predominantly cubic character. This is not unexpected since the differences in positions of the zerophonon lines of $\mathrm{Ce}$ in various sites in Na-co-doped and Na-free crystals of $\mathrm{CaF}_{2}$, studied by Hollingsworth and McClure [7], were not large (usually much less than $100 \mathrm{~cm}^{-1}$ ).

Both d-bands are missing in the "slow" spectrum measured with larger delay (150 ns). This is to be expected since the Ce-emission that follows direct excitation into one of the d-bands decays with the radiative lifetime of about $30 \mathrm{~ns}$ [5]. More unusual is the third band, C, positioned between 127 and $140 \mathrm{~nm}$, below the bandgap and the first exciton peak in $\mathrm{BaF}_{2}$ at $10 \mathrm{~K}(117 \mathrm{~nm}$ and $124 \mathrm{~nm}$ respectively, [8]), which also generates fast Ce-emission but is unlikely to be due to any direct transition at $\mathrm{Ce}^{3+}$ ion. The seemingly obvious interpretation involving free excitons is unlikely since the first excitonic peak corresponds to the reflection peak and, consequently, a dip in the excitation spectrum at about $120 \mathrm{~nm}$. We also note that excitation into the first excitonic peak favors a well known excitonic emission (self-trapped exciton, STE, see e.g. [5]). These observations explain a steep decrease of the C-band at the short wavelength side and emphasize the need to invoke a different interpretation of the C-band itself.

We propose that the $\mathrm{C}$-band is due to an unrelaxed state of the exciton bound to $\mathrm{Ce}^{3+}$ ion. The energy transfer to one of $5 \mathrm{~d}$ states of the $\mathrm{Ce}^{3+}$ ion, facilitated by some preceding lattice relaxation, is likely to be very efficient and fast, as observed in the experiment.

In addition to the $121.4 \mathrm{~nm}$ dip we note also three other dips; at $96.8,85.2$ and $72.8 \mathrm{~nm}$. These dips correspond to other reflection peaks reported earlier [8].

A good VUV sensitivity is expected and desired for scintillation detector material. Although, in $\mathrm{BaF}_{2}$ :Ce the emission is due almost exclusively to Ce ions and not to STEs and VUV response is good but, unfortunately, it is characterized by long decay times [5]. Despite a significant fast component, a large fraction of energy transferred to Ce-ions by this channel (most likely via free electron-hole pairs) is intercepted by traps responsible for slow components in the scintillation time profile of $\mathrm{BaF}_{2}: \mathrm{Ce}$.

The earlier Superlumi measurements of emission and excitation spectra of $\mathrm{Er}^{3+}$ in $\mathrm{BaF}_{2}$ have already been reported for the $0.2 \mathrm{~mol} \%$ Er sample [9]. Since there was no difference between "fast" and "slow" time window spectra, in Fig. 2 we show some of the recently measured time-integrated excitation spectra of the $\mathrm{Er}^{3+}$ VUV and VIS emissions from a different $\mathrm{BaF}_{2}$ sample doped with $0.05 \mathrm{~mol} \%$ Er.

The red and blue lines show the spectrum measured at two wavelengths (163.5 and $165 \mathrm{~nm}$, res. $1.1 \mathrm{~nm}$, step 0.1 and $0.01 \mathrm{~nm}$, respectively) of the VUV emission band peaking at $163.5 \mathrm{~nm}$. The black line shows the spectrum measured at $547 \mathrm{~nm}$ corresponding to the well known "green" emission from Er $\left({ }^{4} S_{3 / 2}\right.$ to $\left.{ }^{4} I_{15 / 2}\right)$. While d-bands, dominating the 163.5 and $165 \mathrm{~nm}$ spectra, are missing, there is a strong band in the $547 \mathrm{~nm}$ spectrum, centered at about $131 \mathrm{~nm}$ that clearly resembles the C-band in the excitation spectrum of Ce emission. It seems reasonable, therefore, to assume that this band is due to the Er-bound 


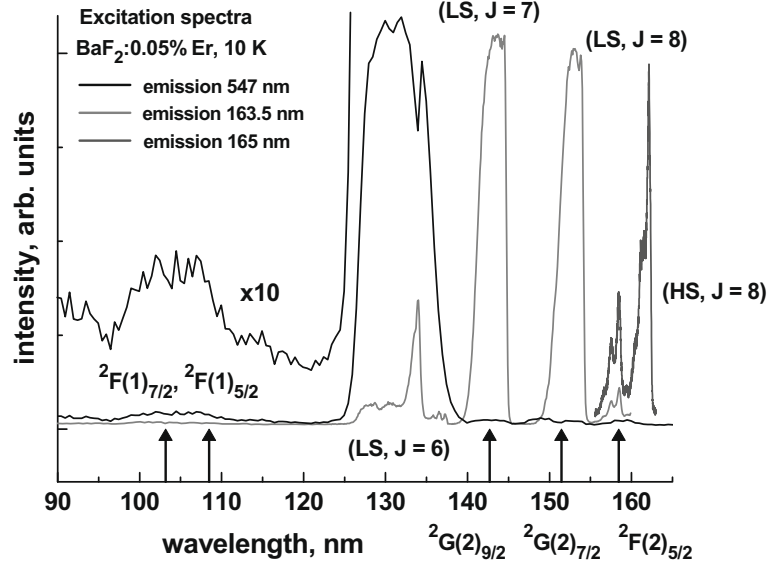

Fig. 2. Time integrated excitation spectra of $\mathrm{Er}^{3+}$ emissions in $\mathrm{BaF}_{2}: 0.05 \mathrm{~mol} \% \mathrm{Er}$ at $10 \mathrm{~K}$. The spectra have been corrected using a salicylate standard. Resolution was $0.32 \mathrm{~nm}$. Black line, emission wavelength at $547 \mathrm{~nm}$, step $0.5 \mathrm{~nm}$, red and blue lines emission wavelengths set at 163.5 and $165 \mathrm{~nm}$, step was set at 0.5 and $0.01 \mathrm{~nm}$ respectively. Black arrows indicate positions of the relevant $4 \mathrm{f}^{11}$ levels. For details, see text. (For interpretation of the references to colour in this figure legend, the reader is referred to the web version of this article.)

exciton which can transfer its energy to any of the lower lying $4 \mathrm{f}^{11}$ levels (like ${ }^{4} \mathrm{~S}_{3 / 2}$ ) but large relaxation prevents energy transfer to the higher lying levels of both $4 \mathrm{f}^{11}$ and $4 \mathrm{f}^{10} 5 \mathrm{~d}$ configurations [9].

It is interesting to note a peak and a corresponding indentation at $133.9 \mathrm{~nm}$ in the red and black line spectra shown in Fig. 2. This observation strongly suggests that there are two separate processes providing excitation of VUV and VIS Er emissions that compete for the excitation photons. We identify these two processes as a direct $\mathrm{Er}^{3+} \mathrm{d}$-excitation leading mostly to d-f VUV emission and an Er-bound exciton exciting $\mathrm{f}-\mathrm{f}$ VIS emission of $\mathrm{Er}^{3+}$ [9].

None of the spectra shown in Fig. 2 reveals a significant VUV sensitivity suggesting a lack of good response to ionizing radiation. A very weak VUV signal at $547 \mathrm{~nm}$ is, as revealed by the emission spectrum under VUV excitation (not shown), mostly due to the STE emission and not to emission from Er ions.

The $163.5 \mathrm{~nm}$ emission band, dominating the spectrum shown in Fig. 3, is generated by the slow, spin forbidden optical transition between the lowest energy, high spin (HS) d-level of the $4 \mathrm{f}^{10} 5 \mathrm{~d}$ $\mathrm{Er}^{3+}$ configuration [10] and the ground state of the $4 \mathrm{f}^{11} \mathrm{Er}^{3+}$ config-

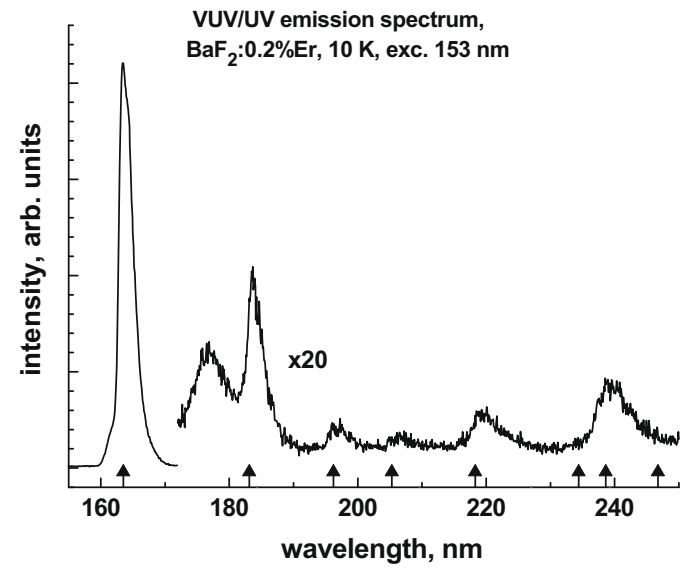

Fig. 3. Uncorrected time integrated VUV/UV emission spectrum of $\mathrm{BaF}_{2}: 0.05 \mathrm{~mol} \% \mathrm{E}$ at $10 \mathrm{~K}$. Excitation was $153 \mathrm{~nm}$ (maximum of LS band). Resolution was $1.1 \mathrm{~nm}$, step $0.1 \mathrm{~nm}$. To expose fine details part of the spectrum was multiplied by a factor of 20 . Arrows indicate calculated positions of d-bands corresponding to transitions from the lowest HS d-level and terminating at consecutive lowest states of the $4 \mathrm{f}^{11}$ configuration. uration, ${ }^{4} \mathrm{I}_{15 / 2}$. The corresponding band in the excitation (absorption) spectrum peaks at about $162.1 \mathrm{~nm}$ (blue line in Fig. 2). The two much stronger bands at 153 and $143.2 \mathrm{~nm}$ and the third competition-distorted band at about $133.9 \mathrm{~nm}$ produce the same VUV and UV emissions and are due to the spin allowed transitions from the ground $4 \mathrm{f}^{11}$ state, ${ }^{4} \mathrm{I}_{15 / 2}$, to higher energy states of the $4 \mathrm{f}^{10} 5 \mathrm{~d}$ configuration [9]. Although there is some similarity between bands at 133.9 and $162.1 \mathrm{~nm}$ which led us previously to a different assignment of the $133.9 \mathrm{~nm}$ band [9] we note that this band is strongly distorted by a competition which makes any comparisons very difficult. Also, the energy difference between the 133.9 and $153 \mathrm{~nm}$ bands is much lower than the $15200 \mathrm{~cm}^{-1}$ 10Dq lower bound value estimated from the Ce-excitation spectrum (Fig. 1).

Despite that Ce and Er ions most likely occupy the same site and should experience the same crystal field, the number and relative positions of the high lying energy levels involving d-electrons, represented by bands in Figs. 1 and 2, are very different. Clearly the crystal field splittings of the fivefold degenerate d-level that fully explain the excitation spectrum of $\mathrm{Ce}$, in no way match the structure observed for the spin-allowed bands in Er.

The origin of this structure has been proposed by van Pieterson et al. [11] to come from the excitation of the $4 \mathrm{f}^{10}$ core left behind by an electron moved to the lowest energy $5 \mathrm{~d}$ orbital. The transition energies are therefore increased by energies corresponding to transitions between the consecutive states $\left({ }^{5} \mathrm{I}_{8},{ }^{5} \mathrm{I}_{7},{ }^{5} \mathrm{I}_{6}\right)$ of the $4 \mathrm{f}^{10}$ configuration $\left(\mathrm{Ho}^{3+}\right.$ ion), producing higher lying bands in the excitation spectrum of the $\mathrm{Er}^{3+} \mathrm{d}-\mathrm{f}$ emission.

The bands shown in Fig. 2 have been labeled by the J-values corresponding to the consecutive $4 \mathrm{f}^{10}$ states reached by a given transition. The lowest states of the $\mathrm{Er}^{3+} 4 \mathrm{f}^{10} 5 \mathrm{~d}$ configuration must have a total spin $S$ equal to $5 / 2$ or $3 / 2$ hence we have used the "LS" label to designate a spin $3 / 2$ level and a spin-allowed transition band (the ground state is ${ }^{4} \mathrm{I}_{15 / 2}$, spin $3 / 2$ ) while "HS" designates a spin $5 / 2$ level and a spin-forbidden transition band.

The arrows in Fig. 2 indicate energies of $4 \mathrm{f}^{11}$ levels calculated by Piatkowski [12] in the framework of the free ion approximation by diagonalization of the appropriate energy matrix. M.F. Reid's fshell empirical programs were used to evaluate the energy parameters. The experimental values of only low energy levels (up to $35,000 \mathrm{~cm}^{-1}$ ) from the absorption spectrum of $\mathrm{BaF}_{2}: 0.2 \mathrm{~mol} \% \mathrm{Er}$ were included in the calculations which provided, nevertheless, theoretical energies for all the states of the $4 \mathrm{f}^{11}$ configuration. The root mean square deviation between the experimental and calculated energies was $90 \mathrm{~cm}^{-1}$.

Of the five states, ${ }^{2} \mathrm{~F}(1)_{7 / 2},{ }^{2} \mathrm{~F}(1)_{5 / 2},{ }^{2} \mathrm{G}(2)_{9 / 2},{ }^{2} \mathrm{G}(2)_{7 / 2}$ and ${ }^{2} \mathrm{~F}(2)_{5 / 2}$, only one, ${ }^{2} \mathrm{~F}(2)_{5 / 2}$, fits some sharp line features in the spectra. None of the remaining four states is expected to play any role. In particular the ${ }^{2} \mathrm{G}(2)_{J}$ levels overlap the centers of strong allowed d-bands and any contribution from them is highly unlikely. Even if there is some weak absorption into one of those levels, it must be immediately depleted nonradiatively into the overlapping d-level as the configuration coordinate parabola of the d-level intersects the f-level parabola near its minimum.

In Fig. 3 we show time-integrated emission spectrum under $153 \mathrm{~nm}$ excitation (d-band) at $10 \mathrm{~K}$. The spectrum has not been corrected for spectral sensitivity of the set-up. To aid presentation two different vertical scales have been used to expose fine details of the spectrum. The arrows indicate calculated positions of bands corresponding to transitions originating at the lowest d-level and terminating at the lowest levels of the $4 \mathrm{f}^{11}$ configuration. The agreement between experimental and calculated positions of bands in the VUV range of wavelengths is reasonably good.

In Fig. 4 we present a time-integrated excitation spectrum of the ${ }^{4} \mathrm{~S}_{3 / 2}-{ }^{4} \mathrm{I}_{15 / 2}$ green $\mathrm{Er}$ emission at $10 \mathrm{~K}$ for the sample of $(\mathrm{Ba}$,$\mathrm{La} \mathrm{F}_{2}: 0.2 \mathrm{~mol} \%$ of Er. The spectrum resembles the spectrum measured for VUV emissions in Er-activated $\mathrm{BaF}_{2}$ and shown in Fig. 2 


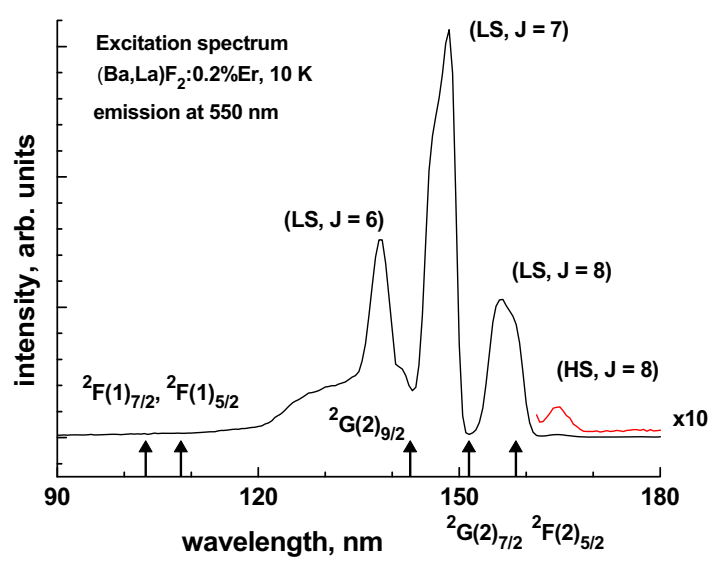

Fig. 4. Time integrated excitation spectrum of $\mathrm{Er}$ emission at $550 \mathrm{~nm}$ in (Ba,La) $\mathrm{F}_{2}: 0.2 \mathrm{~mol} \% \mathrm{Er}$ at $10 \mathrm{~K}$. Resolution was $0.32 \mathrm{~nm}$, step $0.5 \mathrm{~nm}$. The red line shows part of the spectrum multiplied by 10 to expose the low intensity HS band peaking at $165 \mathrm{~nm}$. Arrows indicate calculated positions of $4 \mathrm{f}^{11}$ levels. (For interpretation of the references to colour in this figure legend, the reader is referred to the web version of this article.)

(red and blue lines). The red trace shows the same spectrum in the range of the spin-forbidden transition to the lowest (HS, $J=8$ ) $4 \mathrm{f}^{10} 5 \mathrm{~d}$ state multiplied by a factor of 10 in order to expose a weak spin-forbidden band on the short wavelength side of the first spinallowed band peaking at $157 \mathrm{~nm}$. We note that positions of all the bands in $(\mathrm{Ba}, \mathrm{La}) \mathrm{F}_{2}$ are shifted in comparison to $\mathrm{BaF}_{2}$; the $(\mathrm{HS}, J=8)$ band peaks at 164.6 instead of $162.1 \mathrm{~nm}$, the (LS, $J=8)$ band peaks at 157 instead of $153 \mathrm{~nm}$, the (LS, $J=7$ ) band peaks at 148.5 instead of $143.2 \mathrm{~nm}$ and the (LS, $J=6$ ) band peaks at 138.1 instead of $133.9 \mathrm{~nm}$. The shift of d-bands reflects the change in crystal field brought about by a large La co-doping; the resultant massive presence of compensating fluorine interstitial ions is expected to change (increase) the low symmetry crystal field component. The low symmetry crystal field component is responsible, together with the spin-orbit coupling, for splitting of the $5 \mathrm{~d}(\mathrm{e})$ levels. For larger splittings we expect a lower transition energy, hence a red shift of relevant absorption bands, as observed experimentally.

We note that shifts of d-levels results in the change of relative positions of $4 \mathrm{f}^{11}$ levels. Calculated positions of the five relevant $4 \mathrm{f}^{11}$ levels are shown by arrows. We observe that the two ${ }^{2} \mathrm{G}(2)_{J}$ levels no longer overlap the maxima of LS bands but fall between them and the ${ }^{2} \mathrm{~F}(2)_{5 / 2}$ level, which, in $\mathrm{BaF}_{2}$, was positioned half way between the LS and HS bands, now overlaps the LS band.

In Fig. 5 we show the time resolved VUV/UV emission spectra of (Ba,La) $\mathrm{F}_{2}$ :Er under excitation into the $157 \mathrm{~nm}$, spin-allowed band where the black line presents "fast", and a red line - "slow" time window spectra. There is also shown a time-integrated emission spectrum under excitation into the $165 \mathrm{~nm}$ spin-forbidden band. The "slow" spectrum clearly demonstrates that most of the emission is fast (the decay time is $46 \mathrm{~ns}$ ) but there is also a slowly decaying band at $170 \mathrm{~nm}$ at the longer wavelengths side of the dominant "fast" band at $162.3 \mathrm{~nm}$ that becomes visible for longer delays. The "slow" band at $170 \mathrm{~nm}$ dominates the spectrum under the $165 \mathrm{~nm}$ excitation corresponding to the spin-forbidden transition to the lowest HS state of the $4 \mathrm{f}^{10} 5 \mathrm{~d}$ configuration, as expected.

We observe that in both materials, $\mathrm{BaF}_{2}: \mathrm{Er}$ and $(\mathrm{Ba}, \mathrm{La}) \mathrm{F}_{2}: \mathrm{Er}$, the VUV emission resulting from direct excitation into the lowest energy (HS, $J=8$ ) level, is slow, as expected. This is no longer the case for higher d-level excitations. In $\mathrm{BaF}_{2}$ :Er excitation into the $153 \mathrm{~nm}$ LS band is followed by relaxation and slow emission from the (HS, $J=8$ ) level. In (Ba,La) $F_{2}:$ Er excitation into the LS band at $157 \mathrm{~nm}$ band corresponding to the (LS, $J=8$ ) level, is followed by a fast, spin-allowed emission from the same level with almost no relaxation to the lower $(\mathrm{HS}, J=8)$ level.

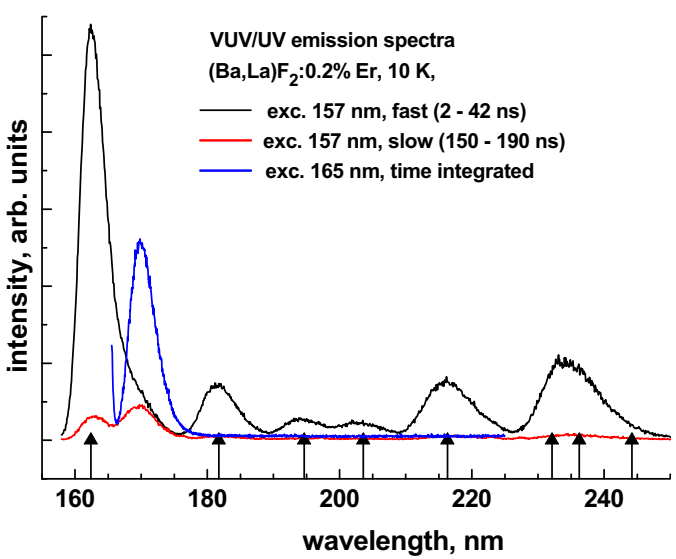

Fig. 5. Uncorrected VUV/UV emission spectra of $(\mathrm{Ba}, \mathrm{La}) \mathrm{F}_{2}: 0.2 \mathrm{~mol} \% \mathrm{Er}$ at $10 \mathrm{~K}$. Black and red lines present time resolved emission spectra under $157 \mathrm{~nm}$ (LS band) excitation. Resolution was $1.1 \mathrm{~nm}$, step $0.1 \mathrm{~nm}$. Blue line presents time integrated emission spectrum under $165 \mathrm{~nm}$ excitation (HS band). Resolution was $1.1 \mathrm{~nm}$, step $0.05 \mathrm{~nm}$. Arrows indicate calculated positions of d-bands corresponding to transitions originating from the lowest LS level and should be compared to positions of the spin-allowed "fast" bands (black line). (For interpretation of the references to colour in this figure legend, the reader is referred to the web version of this article.)

The obvious conclusion must be that relaxation between the lowest LS and HS levels is strongly enhanced when the ${ }^{2} \mathrm{~F}(2)_{5 / 2}$ level is positioned between them. This is the case in $\mathrm{BaF}_{2}$ but in $(\mathrm{Ba},-$ La) $\mathrm{F}_{2}$, where stronger lower symmetry crystal field component shifts the lowest d-levels downwards, is not.

In Fig. 6 we show a simple configuration coordinate model for the three relevant electronic states of the $\mathrm{Er}^{3+}$ ion in $\mathrm{BaF}_{2}$ and $(\mathrm{Ba},-$ La) $\mathrm{F}_{2}, 4 \mathrm{f}^{11}{ }^{2} \mathrm{~F}(2)_{5 / 2}, 4 \mathrm{f}^{10} 5 \mathrm{~d}(\mathrm{HS}, J=8)$ and $4 \mathrm{f}^{10} 5 \mathrm{~d}(\mathrm{LS}, J=8)$. We assume that all the $4 \mathrm{f}^{11}$ states, in particular the ground state ${ }^{4} \mathrm{I}_{5 / 2}$ (not shown) and the ${ }^{2} \mathrm{~F}(2)_{5 / 2}$ state (black solid line) assume equilibrium positions for the same value of the configuration coordinate, namely zero. The equilibrium positions of the two lowest energy $4 \mathrm{f}^{10} 5 \mathrm{~d}$ state parabolas were chosen to fit experimental values of appropriate transitions from absorption (excitation) and emission spectra. From the figure it is clear that in $\mathrm{BaF}_{2}$ the ${ }^{2} \mathrm{~F}(2)_{5 / 2}$ parabola

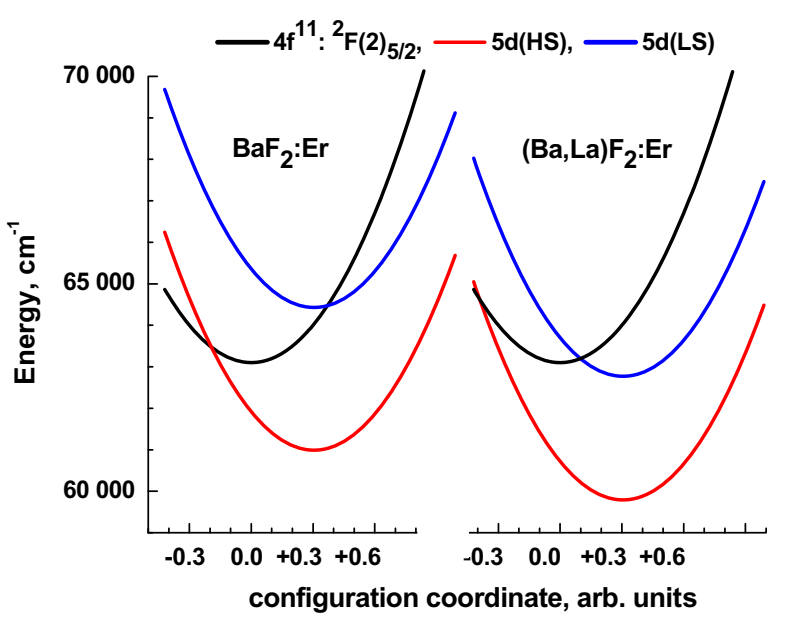

Fig. 6. Configuration coordinate diagrams showing energies of two $4 \mathrm{f}^{10} 5 \mathrm{~d}$ states,

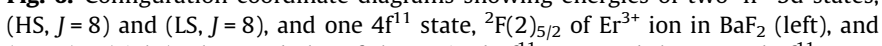
$(\mathrm{Ba}, \mathrm{La}) \mathrm{F}_{2}$ (right). The parabolas of the excited $4 \mathrm{f}^{11}$ state and the ground $4 \mathrm{f}^{11}$ state, ${ }^{4} \mathrm{I}_{15 / 2}$, assume minimum energy at zero. The equilibrium positions of d-states parabolas have been shifted to fit experimental transition energies from excitation and emission spectra. Note the shifts of d-level electronic energies responsible for relative position of the $4 \mathrm{f}^{11}$ level. (For interpretation of the references to colour in this figure legend, the reader is referred to the web version of this article.) 
intersects the two $4 \mathrm{f}^{10} 5 \mathrm{~d}$ parabolas in such a way that it may contribute to the nonradiative transition (relaxation) between them. In $(\mathrm{Ba}, \mathrm{La}) \mathrm{F}_{2}$ situation is different and nonradiative relaxation between the two d-levels must be, and apparently is, much slower

\section{Summary and conclusion}

In this paper we have presented selected examples illustrating the significance of VUV spectroscopy in studies of wide bandgap materials activated with rare earth ions.

VUV/UV excitation spectra help to identify and evaluate various channels of energy transfer from the host to the emitting ion. We have pointed out that, in addition to direct excitation of the ion, there is also a fast and efficient channel involving excitons bound to the ion. Direct optical excitation into the band corresponding to this process is followed by fast and efficient emission with no delay and no rise time. On the contrary, free excitons are unlikely to transfer their energy to the ions creating, instead, self-trapped excitons (STE).

We note that for the rare earth bound exciton a significant fraction of energy is lost to lattice relaxation so that, consequently, no VUV/UV emission can be excited and observed.

The VUV excitation with photon energies exceeding bandgap energy generates free electron-hole pairs that may be consecutively trapped and recombined in the vicinity of the rare earth ion with transfer of recombination energy to the ion. Since the intermediate step in this process involves the rare earth bound exciton no high energy $4 \mathrm{f}^{n-1} 5 \mathrm{~d}$ or $4 \mathrm{f}^{n}$ levels can be excited. Consequently the $\mathrm{Ce}^{3+}$ ion with its near UV d-f transition stands a chance to be good radiative recombination center while $\mathrm{Er}^{3+}$, emitting in the VUV/UV, does not.

The VUV excitation spectra provide, therefore, a convenient test revealing how good a given material is in response to ionizing radiation. This is important in applications involving detection of gamma and/or X-ray radiation.

We have demonstrated that comparison between excitation spectra of light (here $\mathrm{Ce}$ ) and heavy (Er) rare earth ions reveals interesting and important differences in their electronic structure.

The excitation spectra of heavy ions reveal additional weak bands corresponding to spin-forbidden transitions from the ground state to the exchange split HS states of the $4 \mathrm{f}^{n-1} 5 \mathrm{~d}$ configuration. Unlike for the $\mathrm{Ce}^{3+}$ ion, for which the structure of excited states is fully described by the crystal field split d-level, in $\mathrm{Er}^{3+}$ the excited states of the $4 \mathrm{f}^{10} 5 \mathrm{~d}$ configuration are determined by excited states of the d-electron as well as of the $4 \mathrm{f}^{10}$ configuration, changing the number and positions of bands in the excitation (absorption) spectrum.
We have also demonstrated that studies of VUV emission and excitation spectra can provide important information concerning radiative and nonradiative relaxation of highly excited rare earth ions. In particular we have demonstrated that nonradiative relaxation between consecutive LS and HS energy levels of the $\mathrm{Er}^{3+} 4 \mathrm{f}^{10} 5 \mathrm{~d}$ configuration may be relatively slow. In consequence it is possible to observe fast and efficient emission from the LS level as in Er activated $(\mathrm{Ba}, \mathrm{La}) \mathrm{F}_{2}$. In $\mathrm{BaF}_{2}: \mathrm{Er}$, where d-levels are shifted because of modified crystal field, fast nonradiative relaxation from the LS to HS level is facilitated by a $4 \mathrm{f}^{11}{ }^{2} \mathrm{~F}(2)_{5 / 2}$ level located almost exactly halfway between them. Consequently the VUV emission of $\mathrm{BaF}_{2}$ is dominated by slow, spin-forbidden transition between the HS level of the $4 \mathrm{f}^{10} 5 \mathrm{~d}$ configuration and the ground state $4 \mathrm{f}^{11} 4 \mathrm{I}_{15 / 2}$.

\section{Acknowledgments}

The crystals used in this study have been offered by late Prof. Alex Lempicki of Boston University who supported me and the group from N. Copernicus University for many years. I also gratefully acknowledge Prof. Georg Zimmerer and Dr Gregory Stryganyuk from Hasylab for help and hospitality during Superlumi experiments, Prof. M.F. Reid of Canterbury University, Christchurch, New Zealand for his f-shell empirical programs to calculate lanthanide ions $4 \mathrm{f}^{11}$ energy levels. I am grateful to Dawid Piatkowski who actually performed these calculations and to Sebastian Janus, Robert Theis and Kinga Jastak, who took part in experiments at Hasylab. I also thank DESY and EC for financial support during Superlumi experiments under Contract RII3-CT-2004-506008 (IASFS).

\section{References}

[1] R.T. Wegh, A. Meijerink, R.J. Lamminmäki, J. Hölsa, J. Lumin. 87-89 (2000) 1002-1004.

[2] P.S. Peijzel, A. Meijerink, R.T. Wegh, M.F. Reid, G.W. Burdick, J. Solid State Chem. 178 (2005) 448

[3] R.T. Wegh, E.V.D. van Loef, G.W. Burdick, A. Meijerink, Mol. Phys. 101 (2003) 1047.

[4] G. Zimmerer, Nucl. Instrum. Methods Phys. Res. A 308 (1991) 178.

[5] A.J. Wojtowicz et al., Hasylab Annual Report 1998, Hamburg 1998: <http:// hasyweb.desy.de/science/annual_reports/1998/part1/contrib/22/521.pdf>.

[6] W.J. Manthey, Phys. Rev. B8 (1973) 4086

[7] G.J. Hollingsworth, D.S. McClure, Phys. Rev. B48 (1993) 13280.

[8] J.W. Hodby, in: W. Hayes (Ed.), Crystals with the Fluorite Structure, Clarendon Press, Oxford, 1974, p. 24

[9] A.J. Wojtowicz, Opt. Mater. 31 (2009) 474-478.

[10] (a) R.T. Wegh, H. Doker, A. Meijerink, Phys. Rev. B57 (1998) R2025; (b) R.T. Wegh, A. Meijerink, Phys. Rev. B60 (1999) 10820.

11] (a) L. van Pieterson, M.F. Reid, A. Meijerink, Phys. Rev. Lett. 88 (2002) 067405 (b) L. van Pieterson, M.F. Reid, G.W. Burdick, A Meijerink, Phys. Rev, B65 (2002) 045114

[12] A.J. Wojtowicz, S. Janus, D. Piatkowski, presented at ICL 08, Lyon, submitted to J. Lumin. 